\title{
Novel Case of Metastatic Testicular Immature Teratoma Invading the Duodenum
}

\author{
Timothy V. Johnson ${ }^{1}$, Adeboye O. Osunkoya ${ }^{1,2}$, Keith Delman ${ }^{3,5}$, Deborah A. Baumgarten ${ }^{4}$ and \\ Viraj A. Master ${ }^{*}, 1,5$
}

Departments of ${ }^{1}$ Urology, ${ }^{2}$ Pathology, ${ }^{3}$ Surgery, ${ }^{4}$ Radiology and ${ }^{5}$ Winship Cancer Institute, Emory University, Atlanta, GA, USA

\begin{abstract}
We present the first report of an immature teratoma arising from a germ cell testicular cancer invading the duodenum. While other seminomatous and non-seminomatous germ cell tumors are known to metastasize to the duodenum, this is the first such case involving an immature teratoma directly invading the duodenum from a metastatic retroperitoneal mass.
\end{abstract}

Keywords: Testis cancer, teratoma, duodenum.

\section{CASE REPORT}

A 26-year-old male underwent radical inguinal orchiectomy at an outside facility for a pT1 Nx Mx mixed nonseminomatous germ cell tumor (65\% mature teratoma, $30 \%$ yolk sac, $15 \%$ embryonal). Preoperative tumor markers showed ( $\alpha$-fetoprotein (AFP), $2096 \mathrm{ng} / \mathrm{mL}$ and $\beta$-Human chorionic gonadotropin (B-HCG), $139 \mathrm{mIU} / \mathrm{ml}$ ). Postoperative tumor markers normalized AFP $3.6 \mathrm{ng} / \mathrm{mL}$ and B-HCG $<2 \mathrm{mIU} / \mathrm{ml}$ ) and CT scans of the chest and abdomen showed no signs of metastasis. Eighteen months later, the patient presented to our facility with progressive nausea and vomiting. Abdominal CT scan (Fig. 1) and CT-guided biopsy revealed a $9.4 \mathrm{~cm}$ retroperitoneal tumor consistent with teratomatous elements of a mixed germ cell tumor. The duodenum proximal to the mass was dilated, indicating an element of obstruction. In retrospect, there was discontinuity of the duodenal wall indicating possible invasion by the mass (Fig. 1). Four months later, following four cycles of etoposide and cisplatin, the tumor shrunk to $5.8 \mathrm{~cm}$. At this point, there were punctuate calcifications within the mass; the mass remained intimately associated with the anterior aorta and the undersurface of the third portion of the duodenum with interval clinical relief of the duodenal obstruction (Fig. 2). The patient's tumor markers remained within normal limits (AFP, $<5 \mathrm{ng} / \mathrm{mL}$ and B-HCG, $<1$ $\mathrm{mIU} / \mathrm{ml}$ ). The patient underwent a retroperitoneal lymph node dissection and mass removal. Intraoperatively, the mass was easily dissected off the aorta and inferior vena cava but was found to be directly invading the duodenum, requiring resection of a portion of the duodenal wall to achieve negative surgical margins. Pathology revealed a mass consisting of $90 \%$ mature teratoma and $10 \%$ immature teratoma (Fig. 3), with invasion of the wall of duodenum, as confirmed by two subspeciality genitourinary pathologists. His clinical course was complicated by a readmission within the first 30 post-operative days, due to nausea and vomiting,

*Address correspondence to this author at the Department of Urology, 1365 Clifton Road NE, Atlanta, GA 30322, USA; Tel: 404-778-3859;

E-mail: vmaster@emory.edu
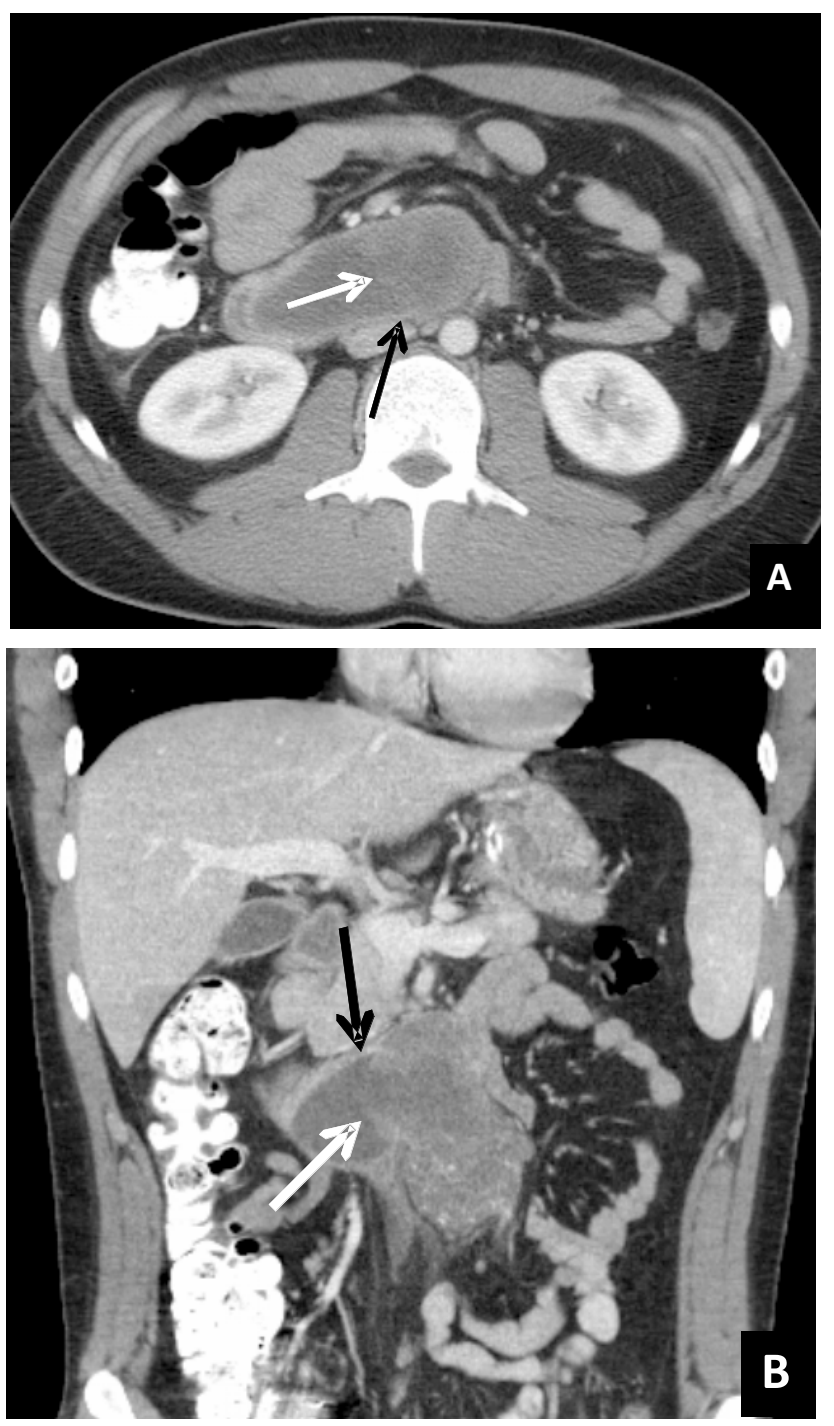

Fig. (1). A, axial enhanced CT image and B, coronal enhanced CT reconstruction demonstrate invasion of the duodenum by metastatic adenopathy from testicular cancer. The duodenal wall (black arrows) is disrupted by tumor (white arrows). 

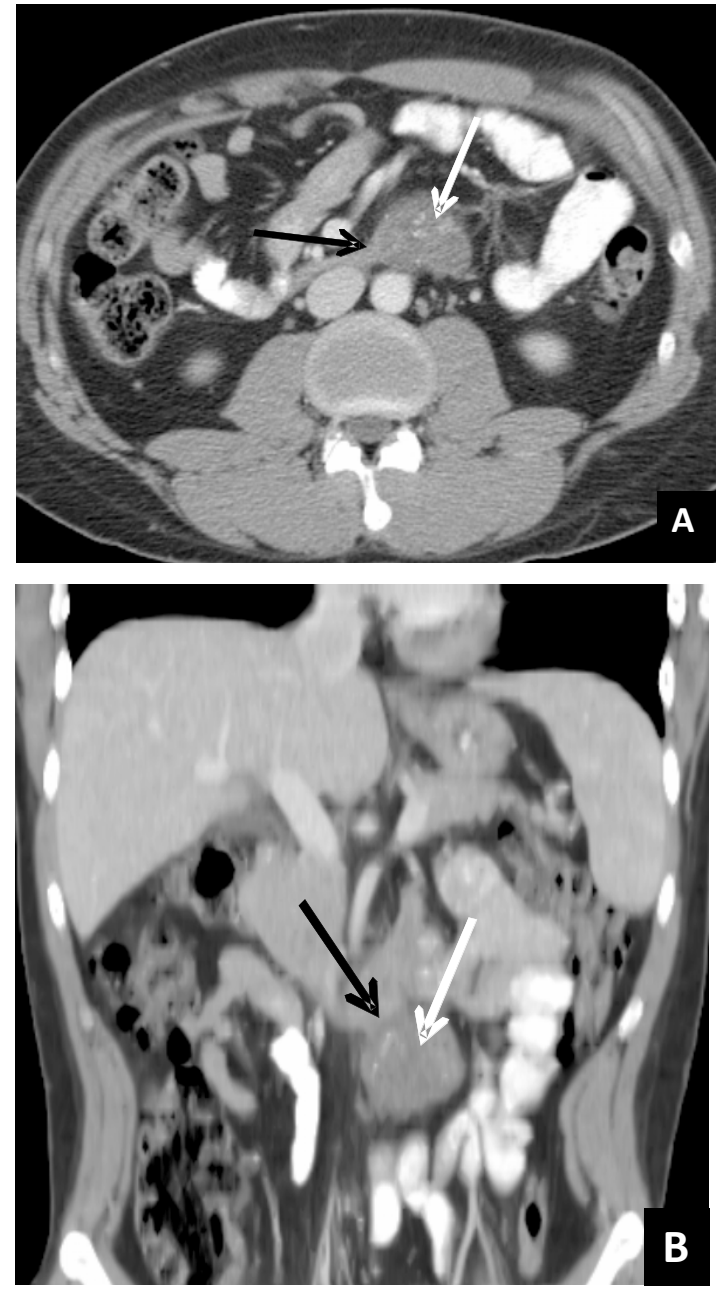

Fig. (2). After treatment, A, axial enhanced CT image and B, coronal enhanced CT reconstruction show interval reduction in mass size and development of punctate calcifications within the mass (white arrow). The duodenum (black arrow) is splayed around the mass but no longer dilated. necessitating several days of nasogastric tube drainage, with subsequent resolution. Three years post-operatively, the patient is fully recovered without evidence of recurrent disease. and being closely monitored both serologically and radiologically.

\section{DISCUSSION}

Germ-cell tumors (GCTs) are the most common type of cancer in men age 15 to 35 [1]. Of GCTs that originate in the testis, $60 \%$ are nonseminomatous (NSGCTs) [1]. NSGCTs are histopathologically heterogeneous, and commonly consist of varying percentages of embryonal cell carcinoma, yolk sac tumor, choriocarcinoma, and teratoma $[1,2]$. While teratomatous elements are found in $55-85 \%$ of all NSGCTs in adults, only $2-6 \%$ of are pure teratomas [2]. Pure teratomas can be further subdivided by the sexual development of the patient: prepubertal and postpubertal testicular teratomas [2]. Neither prepubertal nor postpubertal teratomas are associated with increased $\alpha$-fetoprotein or $\beta$ Human chorionic gonadotropin tumor markers, and they are both composed of more than one element from different germ layers (endoderm, mesoderm, and ectoderm) [2, 3].

Though teratomas in prepubertal males are benign, teratomas in postpubertal males have malignant potential irrespective of whether there are mature or immature components [2, 3]. Among patients with pure postpubertal teratomas, approximately two-thirds will present with advanced metastatic disease, most commonly to the retroperitoneum [2-5]. This presentation typically constitutes a regression of other nonseminomatous germ-cell elements in the testis [2]. Teratomas, as well as other NSGCTs, metastasize via lymphovascular invasion [2].

Typically, advanced teratomas and other NSGCTs, especially in the retroperitoneum, present with back pain due to enlargement and compression. Rarely, NSGCTs can also involve the gastrointestinal tract, resulting in intestinal obstruction, gastrointestinal hemorrhage, and occasionally ulceration of the bowel mucosa [6-9]. However, each of

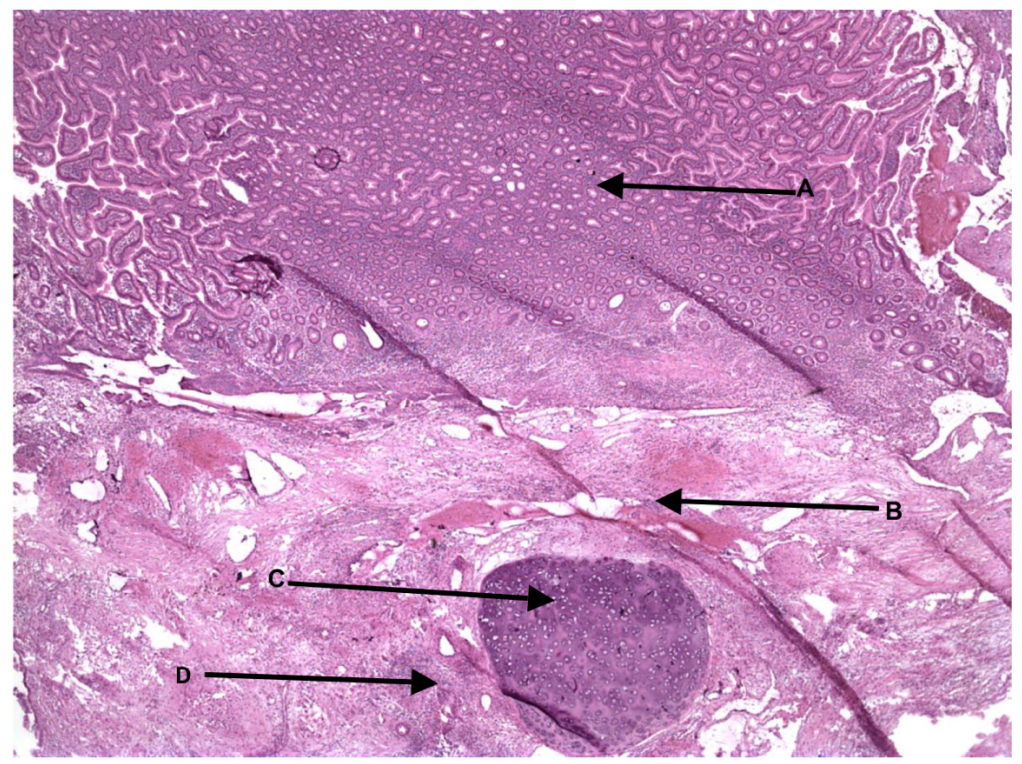

Fig. (3). Pathology slide demonstrating invasion of the duodenum by immature cartilage and adjacent primitive stromal elements (Aduodenal mucosa; B-submucosa; C-Immature cartilage; D-primitive stromal elements). 
these published reports consisted of metastatic seminomatous or mixed NSGCTs. To our knowledge, our patient is the first reported case of the direct gastrointestinal invasion by the immature teratoma. This case highlights, and indeed expands, the important point that any testicular tumors, regardless of histological type, should always be considered as being potentially invasive into the GI tract if it metastasizes to the retroperitoneum. From a surgical standpoint, this may change the bowel prep given to the patient or the choice of preoperative antibiotic, depending on the suspicion for GI tract involvement. The important point of this paper is simply is point out that that multidisciplinary surgical consultation is often highly appropriate in this type of complex patient. The urologist who assumes that retroperitoneal teratomas arising from testicular primary tumors never invade the GI tract would not be prepared for the intraoperative need for GI tract surgery, and the need for surgical oncology involvement. Consultation with a general surgeon may be necessary before surgery, as well as at the time of surgery. It also suggests our need for increased understanding of the manner in which these tumors metastasize and invade local structures.

\section{REFERENCES}

[1] Flechon A, Rivoire M, Droz JP. Management of advanced germcell tumors of the testis. Nat Clin Pract Urol 2008; 5: 2620-76.

[2] Carver BS, Al-Ahmadie H, Sheinfeld J. Adult and pediatric testicular teratoma. Urol Clin North Am 2007; 34: 245-51.

[3] Simmonds PD, Lee AH, Theaker J M et al. Primary pure teratoma of the testis. J Urol 1996; 155: 939-42.

[4] Leibovitch I, Foster RS, Ulbright TM, et al. Adult primary pure teratoma of the testis. The Indiana experience. Cancer 1995; 75: 2244-50.

[5] Rabbani F, Farivar-Mohseni H, Leon A, et al. Clinical outcome after retroperitoneal lymphadenectomy of patients with pure testicular teratoma. Urology $2003 ; 62:$ 1092-6.

[6] Miocinovic R, Abaza R. Testicular seminoma presenting with duodenal perforation: a case report. J Med Case Rep 2008; 2: 2945.

[7] Plukker JT, Schraffordt KH, Sleijfer DT, et al. Intestinal hemorrhages in patients with a nonseminomatous testicular tumor. Cancer 1991; 68: 2630-2.

[8] Sweetenham JW, Whitehouse JM, Williams CJ, et al. Involvement of the gastrointestinal tract by metastases from germ cell tumors of the testis. Cancer 1988; 61: 2566-70.

[9] Nakamura A, Ikeda Y, Morishita S, et al. Upper gastrointestinal bleeding arising from metastatic testicular tumor. J Gastroenterol 1997; 32: 650-3 